\title{
Pseudomonas two-partner secretion toxin Exolysin contributes to insect
}

\section{killing}

\author{
Viviana Job ${ }^{1}$, Stéphanie Bouillot ${ }^{1}$, Erwan Gueguen ${ }^{2}$, Mylène Robert-Genthon ${ }^{1}$, Peter Panchev ${ }^{1}$, Sylvie \\ Elsen $^{1}$ and Ina Attrée ${ }^{1 *}$
}

${ }^{1}$ Université Grenoble Alpes, Bacterial Pathogenesis and Cellular Responses team, CNRS ERL5261, CEA IRIG-BCI, INSERM UMR1036, Grenoble, France

${ }^{2}$ University of Lyon, Université Lyon 1, INSA de Lyon, CNRS UMR 5240 Microbiologie Adaptation et Pathogénie, Lyon, France

Abstract Pseudomonas chlororaphis is a promising biocontrol agent promoting plant-growth and providing protection against pest insects and phytopathogenic fungi. We have identified in the genome of $P$. chlororaphis PA23 an operon encoding the toxin Exolysin (ExIA) and its outer-membrane transporter, ExIB. We found that $P$. chlororaphis producing ExIA $\left(\operatorname{ExIA}^{P c h}\right)$ is cytotoxic towards murine macrophages and human epithelial cells at $30^{\circ} \mathrm{C}$. P. chlororaphis PA23 provoked shrinkage of epithelial cell, leakage of cytoplasmic components and subsequent cell death. During infection, ExIA ${ }^{\text {Pch }}$ incorporated into epithelial cell membranes within detergent-resistant lipid rafts, suggesting the same mechanisms of cell destruction by pore-formation as reported for $P$. aeruginosa toxin. ExIA ${ }^{P c h}$ was not involved in the capacity of the strain to kill fungi, amoeba or other bacteria. The contribution of ExIA in insecticidal activity of $P$. chlororaphis was evaluated in the wax moth larvae Galleria mallonella and in Drosophila melanogaster flies. The impact of the deletion of a gene encoding exIA homologue was tested in the natural fly pathogen $P$. entonomophila. In both models, the ExIA absence delayed killing, suggesting the contribution of the toxin in bacteria-insect pathogenic interactions. 


\section{Introduction}

Bacteria from the genus Pseudomonas are ubiquitous in nature due to their extraordinary capacities to adapt to diverse niches. Pseudomonas species are mainly associated with plants and animals but also frequently found in the proximity of human activities [1]. P. fluorescens, P. putida and $P$. stutzeri are recognized as beneficial with potential uses in bioremediation, production of secondary metabolites, or in agriculture for the plant-growth promoting activity and biocontrol property (reviewed in [1-4]). To the contrary, P. aeruginosa is a well-known human opportunistic pathogen that causes high-cost health problems notably due to the increasing number of multi-drug resistant strains. The World Health Organization [WHO] classified carbapenem-resistant $P$. aeruginosa in a high priority category of bacterial pathogens for which there is an urgent need to define new antibiotics [5].

$P$. chlororaphis belonging to the group complex of $P$. fluorescens thrives on plants and in diverse soil environments. Several strains of $P$. chlororaphis have been selected as potential biocontrol agents as they possess both pest insecticidal activities and are able to suppress the plant fungal pathogen Sclerotinia sclerotiorum [6-8] that causes the disease called "white mold" in a wide range of plants. The spectrum of secondary metabolites produced by $P$. chlororaphis includes phenazines, pyrrolnitrin, hydrogen cyanide and others $[7,9,10]$. In addition, secreted proteinous macromolecules as chitinases, proteases and toxins participate to $P$. chlororaphis success in phytoprotection and promotion of plant growth [9].

Together with phenotypic studies, the accessibility to several fully sequenced $P$. chlororaphis strains available at Pseudomonas database, www.pseudomonas.com [11], allowed the analysis of their genome content with respect to predicted secreted molecules and toxins. The reference strain $P$. chlororaphis PA23 [12] harbors the gene cluster encoding a toxin, Fit, and the predicted dedicated export machinery identified originally in P. protegens [13]. The Fit toxin is responsible for killing of several important lepidopteran pests, notably Spodoptera littoralis (cotton leafworm) and the diamondback moth Plutella xylostella [14]. P. chlororaphis PA23 genome also harbors some genes potentially encoding type III secretion system (T3SS) components and two loci encoding T6SS. In P. chlororaphis, T6SSs participate to the invasion of a Gram-positive bacteria Bacillus subtilis [15] and may play a role in displacement of the pest insect gut microbiome as recently described for $P$. protegens [16]. Moreover, high- 
molecular weight bacteriocins, named Tailocins, could broaden the killing capacities of $P$. chlororaphis in polymicrobial communities $[17,18]$.

Clinical strains of the human opportunistic pathogen $P$. aeruginosa use either T3SS or twopartner secretion (TPS) system ExIB-ExIA to promote host invasion and infection $[19,20]$. The presence of exIBA operon in $P$. chlororaphis suggested its role in establishing pathogenic relationship with other organisms, prokaryotes or eukaryotes. In this work, we examined the expression and functionality of ExIA from $P$. chlororaphis and showed its contribution in killing of Galleria mellonella larvae upon injection. Moreover, ExIA homologue in P. entonomophila, a fly pathogen [21] was found to play a role in oral toxicity of Drosophila melanogaster flies.

\section{Results and discussion}

\section{P. chlororaphis EYO4_RS20945 and EYO4_RS20950 encode ExIB-ExIA}

The search in databases for the closest homologues of $P$. aeruginosa ExIB $\left(\mathrm{ExIB}^{P a}\right)$ and ExIA $\left(E{ }^{P a}\right)$ revealed two contiguous genes, EYO4_RS20945 and EYO4_RS20950 in P. chlororaphis PA23 sharing homologies with exIBA in several clonal $P$. aeruginosa outliers (www.pseudomonas.com, [11], Figure 1). EY04_RS20945 encodes a 65-kDa outer membrane protein homologue to ExIB, a TpsB partner involved in the export of the cognate TpsA secreted protein. EYO4_RS20950 encodes the predicted 170-kDa TpsA secreted partner with a classical Sec secretion signal peptide at the N-terminus followed by the TPS secretion domain, and six predicted FHA repeats. The predicted EY04_RS20945 and EYO4_RS20950 gene products share $67 \%$ and $62 \%$ identity to $\operatorname{ExIB}^{P a}$ and $\mathrm{ExIA}^{P a}$, respectively. Taking into account the high identity scores and the data presented in this work, we renamed EY04_RS20945 and EY04_RS20950 as $e x I B^{P c h}$ and $e x I A^{P c h}$, respectively. Genomic environment of $e x / B^{P c h}$ and $e x I A^{P c h}$ is different from that found in $P$. aeruginosa strains, with several regulatory proteins encoded upstream and downstream of both operons (Figure 1). Notably, two genes upstream of ex/B ${ }^{\text {Pch }}$ encode enzymes involved in biogenesis of the second messenger c-di-GMP, while two genes downstream of exIA $A^{\text {Pch }}$ encode putative two-component regulator system with a histidine kinase (HK) and a transcriptional factor (TF). The region upstream of ex/B ${ }^{P c h}$ ATG start codon harbors a putative promoter (BPROM) with predicted "-35" and "-10" $\sigma^{70}$-RNA polymerase binding sites. The exIBA locus is present in all $P$. chlororaphis strains reported at the Pseudomonas genome database and the syntheny of the locus is shared by $P$. protegens 
strains (www.pseudomonas.com). We also reanalyzed the region of two genes PSEEN_2176 and PSEEN_2177 in a fly pathogen P. entonomophila that code for a two-partner system homologue to ExIB-ExIA ([22], Figure 1). PSEEN_2176 gene product shares 69\% identity with ExIB $^{P c h}$ and $65 \%$ with ExIB ${ }^{P a}$, while PSEEN_2177 codes for an ExIA like-protein with $47 \%$ and $40 \%$ identity with $\operatorname{ExIA}^{P c h}$ and $\operatorname{ExIA}^{P a}$, respectively. We renamed also these genes product as ExIA ${ }^{P e}$ and ExIB ${ }^{P e}$.

\section{ExIA provides cytotoxic phenotype to $P$. chlororaphis PA23}

Virulent $P$. aeruginosa clinical isolates secreting ExIA (ExIA $\left.{ }^{P a}\right)$ display cytotoxicity on a variety of eukaryotic cells by forming pores in the host plasma membrane leading to altered $\mathrm{Ca}^{2+}$ signaling, cleavage of cell-cell junctions and cell death $[23,24]$. To assess the cytotoxic potential of $P$. chlororaphis on eukaryotic cells, we co-incubated at $30^{\circ} \mathrm{C}$ epithelial A549 cells with P. chlororaphis PA23 ranging from multiplicity of infection (MOI) of 0.1 to 100 , and measured the kinetics of incorporation of the membrane-impermeable, propidium iodide (PI) into DNA (Figure 2A). PI incorporation was detected already with $\mathrm{MOI}$ of 0.1 , meaning 1 bacteria per 10 epithelial cells, and the kinetics progressively increased with higher $\mathrm{MOI}$. To ascertain that the observed cytotoxicity of $P$. chlororaphis PA23 was due to the ExIA protein, we engineered a strain in which a part of the ex/B-exIA operon was deleted (Pch $\triangle$ ex/BA) and measured the kinetics of $\mathrm{Pl}$ incorporation during $3 \mathrm{~h}$ in comparison to $P$. aeruginosa strain IHMA87 and the exIA-isogenic mutant (Pa-exIAmut, $[23,25])$ on macrophages and epithelial cells. The Pch $\triangle$ exIBA strain lost the capacity to kill $\mathrm{J774}$ macrophages and A549 epithelial cells (Figure 2B). We then followed the infection process by video-microscopy. Similar to the timelapse consequences provoked by the ExIA-secreting $P$. aeruginosa, $P$. chlororaphis induced initial epithelial cell rounding, followed by the loss of cytoplasmic content, which can be visualized by the loss of cytoplasmic GFP. The incorporation of PI into the cell nuclei occurred at the later stages of infection process (Figure 3). As ExIA ${ }^{P a}$ is a membrane pore-forming toxin and binds to liposomes and host membranes ([23], V. Job, manuscript in preparation), we tested whether ExIA ${ }^{P c h}$ could be detected within infected cell membranes, and in particular within the lipid rafts, detergent-resistant membrane (DRM) fractions enriched in cholesterol and sphingolipids, that is involved in protein sorting, signaling and interaction with the cytoskeleton. To that end, after scaled-up infection conditions, we prepared and analyzed e 
epithelial cell membranes for presence of ExIA ${ }^{P c h}$ by immunoblotting. Both ExIA ${ }^{P c h}$ and ExIA $A^{P a}$ could be recovered in DRM fractions (Figure 4), indicating the same mechanism of interaction with cell membranes and similar action of those two proteins with host membranes.

\section{ExIA ${ }^{P c h}$ in microbial competition and amoeba killing}

P. chlororaphis is considered as a safe biocontrol agent $[26,27]$ and until recently has been rarely found responsible for human infections [28]. P. chlororaphis is frequently associated with plants, to which it provides protection against diverse phytopathogens, including fungi and insects $[2,29]$. We thus wondered whether ExIA ${ }^{P c h}$ could participate in interactions with other microbes. First, we tested an inhibition of $P$. chlororaphis on the plant fungal pathogens Sclerotinia sclerotiorum and Botrytis cinerea $[6,8]$, using radial inhibition assay on agar PDA plates (Figure 5A). No significant difference in fungus growth inhibition could be observed between PA23 wild-type strain and the exIBA mutant, suggesting that the toxin does not participate in fungicide activity of PA23 under condition tested. We tested also putative implication of ExIA in bacteria-bacteria interactions, using B. subtilis, P. aeruginosa and E. coli. In all three cases, no difference could be detected between the $P$. chlororaphis wild-type and mutant strains.

Second, we wondered if ExIA could influence the growth of eukaryotic organisms such as amoeba. For example, P. aeruginosa PAO1 is capable of killing the amoeba Acanthamoeba castellani with its T3SS [30]. To test whether $P$. chlororaphis could also infect $A$. castellani, the pathogenicity of $P$. chlororaphis toward Acanthamoeba castellani cells was determined by using a simple plating assay [31]. As shown in Fig. 5B, 50 amoebae cells are required to form a plaque on $E$. coli, while 10,000 are necessary to form a plaque on $P$. aeruginosa PAO1. This result is explained by the ability of $P$. aeruginosa to kill amoebas by injecting T3SS cytotoxins [31] while E. coli are grazed. With P. chlororaphis, no difference in plaque formation was observed between wild type and $\triangle$ exIBA. In both cases, 5,000 amoebas were required to form a small plaque. This result is similar to that obtained with $P$. aeruginosa. This experiment does not distinguish between killing and growth inhibition of $A$. castellani. Nevertheless, it suggests that ExIA does not play a role in the interaction with the amoeba in conditions tested. Of note, the interactions between the Pseudomonas and amoeba were done on solid medium and ExIA may not be expressed and/or secreted in this condition. Otherwise, the composition of the 
host membranes between permissible and not permissible cells may be of crucial importance for ExIA function.

\section{ExIA contributes to toxicity towards Galleria mellonella larvae and Drosophila flies}

P. chlororaphis strains possess oral and systemic insecticidal activity [9]. To assess the contribution of Exolysin to insect killing, we used G. mellonella larvae and Drosophila melanogaster flies. When P. chlororaphis PA23 bacteria were injected into hemocoel of the larvae, the mortality could be observed at $18-20 \mathrm{~h}$ post-injection (Figure 6) with typical change in color of the larvae which is directly related to melanization, a larvae's immune response (Figure 6A). The mortality rates were dependent on temperature and number of injected bacteria (data not shown). The absence of Exolysin in the mutant Pch $\triangle$ ex/BA attenuated significantly the mortality of infected larvae (Figure 6B), suggesting the contribution of Exolysin in larvae death. We then set-up experiments to evaluate the toxin contribution in $D$. melanogaster killing by feeding the flies with high doses of bacteria. As a positive control, we used a well-known fly pathogen, $P$. entomophila [21, 32], and we included an isogenic mutant (Pe-exIAmut) with a plasmid insertion within PSEEN2177 after residue 442 codon of ExIA ${ }_{(1-}^{\mathrm{Pe}}$ 1416). As previously reported $[33,34]$, female flies being fed with high doses of $P$. entomophila $\left(O D_{600}=100\right)$ died within 2 and 4 days. Interestingly, the Pe-ex/Amut showed significantly lower killing capacity compared to the $P$. entomophila wild-type strain (Figure 7). When used in the same experimental conditions, $P$. chlororaphis PA23 provoked mortality with delay of several days compared to $P$. entomophila (Figure 7), and the contribution of ExIA was less pronounced, although still significant. Together, these results show that in two different Pseudomonas species the disruption of a gene encoding two-partner secretion toxin ExIA reduced bacterial toxicity toward two different insects showing the role of ExIA in both bacteria-insect pathogenic interactions.

\section{Conclusions}

In this work, we characterized ExIB-ExIA system, originally annotated as a TPS secreting a filamentous hemagglutinin adhesin, in a biocontrol bacteria $P$. chlororaphis. ExIA ${ }^{P c h}$ is 
responsible for $P$. chlororaphis cytotoxicity measured on human epithelial cells and mouse macrophages, with similar kinetics observed with ExIA-producing $P$. aeruginosa. The ExIA protein contributed to $P$. chlororaphis toxicity in Galleria mellonella larvae opening the possibility to modulate ExIA levels and to increase the use of $P$. chlororaphis in management of pest insects. The link of ExIB-ExIA system to known toxins (i.e. Fit toxin, or effectors of T6SS) and secondary metabolites known to be important in bacteria-insect interactions should be characterized. Finally, as the Vfr/cAMP pathway activates the $P$. aeruginosa ex/BA expression, and the promoter of $P$. chlororaphis exIB displays no conserved Vfr DNA-binding site, the regulatory mechanisms governing exIBA operon expression in $P$. chlororaphis will be important to investigate. Finally, our work revealed that ExIA contributes to P. entomophila virulence toward Drosophila flies, which opens the question of ExIA activity in this bacteria and the relationship between ExIA and P. entomophila toxin Monalysin that displays poreforming activity and provokes fly intestinal damage [33].

\section{Materials and Methods}

\section{Bacterial strains, growth conditions and genetic constructions}

P. chlororaphis PA23 [12] was a gift from Terasa de Kievit, University of Manitoba, Canada. $P$. aeruginosa IHMA87 is a urinary tract infection isolate and was previously shown to secrete Exolysin [19]. P. entomophila [32] was obtained from Bruno Lemaitre's lab (EPFL, Switzerland) and the plasmid-insertion mutant in the exIA ${ }^{P e}$ gene homologue, PSEEN2177, was given by Isabelle Vallet-Galey (I2BC-CNRS, Paris, France). The exIAPe mutant was verified by PCR and sequencing. For $P$. chlororaphis PA23 AexIBA (RS20945/950) mutant construct, a 900 bp fragment containing a frameshift fusion of the two genes was synthesized by Genewiz and then subcloned in EcoRI-HindIII of pEXG2, leading to pEXG2-Mut-Pchloro_exIBA. The plasmid was transferred into $P$. chlororaphis by triparental mating using pRK600 as a helper plasmid. For allelic exchange, cointegration events were first selected on LB plates containing rifampicin $(25 \mu \mathrm{g} / \mathrm{ml})$ and gentamicin $(25 \mu \mathrm{g} / \mathrm{ml})$ at $28^{\circ} \mathrm{C}$. Single colonies were then plated on $\mathrm{NaCl}$-free LB agar plates containing $10 \%$ (wt/vol) sucrose to select for the loss of plasmid. The sucrose-resistant strains were checked for gentamicin sensitivity and mutant genotype by PCR. The mutant strains harbor a deletion of $18 \%$ of exIA and $32 \%$ of exIB genes. 
Bacteria were grown in liquid LB medium (Becton Dickinson) at $28-30{ }^{\circ} \mathrm{C}$ (for $P c h$ and $\mathrm{Pe}$ ) or $37^{\circ} \mathrm{C}$ (for $\mathrm{Pa}$ ) with $300 \mathrm{rpm}$ agitation. After overnight incubation, strains were diluted in LB medium to reach optical density measured at $600_{\mathrm{nm}}\left(\mathrm{OD}_{600}\right)$ of 1.0 at $30{ }^{\circ} \mathrm{C}$ or $37{ }^{\circ} \mathrm{C}$, respectively.

\section{Cytotoxicity tests}

Epithelial lung carcinoma cell line A549 (ATCC CCL-185), A549-EGFP [35] and J774 macrophages were grown in DMEM or RPMI medium (Life Technologies) supplemented with $10 \%$ fetal calf serum (Lonza). For cytotoxicity test, cells were seeded at 12,500 cells per well for A549 and 100,000 cells per well for J744 on black $\mu$ clear 96-well plates (Greiner) and used $48 \mathrm{~h}$ later to obtain confluent monolayers. One hour before infection, medium was replaced by DMEM or RPMI (for J774 cells) without phenol red, supplemented with PI (Sigma, $1 \mu \mathrm{M}$ ). Cells were infected at a multiplicity of infection (MOI) of 10 , unless otherwise specified. PI incorporation was followed by fluorescence measuring (excitation $544 \mathrm{~nm} / \mathrm{emission} 590 \mathrm{~nm}$ ) every 10 minutes with Fluoroskan Ascent FL2.5 Microplate Fluorometer (Thermo Corporation), during 10 hours at $30{ }^{\circ} \mathrm{C}$. Data were presented as arbitrary fluorescence units (A.U.) as a function of time.

Imaging of infection by confocal video microscopy

For confocal video microscopy experiments, cells were seeded at 100,000 cells per well on Lab-tek II 8-chambered coverslips (Dutscher Scientific) and used $48 \mathrm{~h}$ later to obtain confluent monolayers. One hour before infection, medium was replaced by DMEM without phenol red and supplemented with $1 \mu \mathrm{M}$ PI. Cells were infected at a $\mathrm{MOI}$ of 10 and immediately observed by confocal video microscopy for $6 \mathrm{~h}$ at $30^{\circ} \mathrm{C}$. Video microscopy was performed on a spinningdisk inverted microscope (Nikon TI-E Eclipse) equipped with an Evolve EMCCD camera. The optical sectioning was performed by a Yokogawa motorized confocal head CSUX1-A1. Images were acquired using an illumination system from Roper Scientific (iLasPulsed) with a CFI Plan Fluor oil immersion objective (40X, N.A. 1.3). Z-series were generated every 5 min using a motorized Z-piezo stage (ASI) by acquiring 10 z-plane images with a step size of $1 \mu \mathrm{m}$. Microscope was controlled with MetaMorph software (Molecular Devices). Temperature, $\mathrm{CO}_{2}$, and humidity control was performed using a chamlide TC system (TC-A, Quorum technologies). Solid-state 491 and 561 nm lasers (iLas, Roper Scientific) and ET 525/50M 
(Chroma) and FF01-605/54 (Semrock) emission filters were used for excitation and emission of EGFP and PI fluorescence, respectively. The cells used for analysis were chosen at the initial step of recording. Analyses were performed by measuring the fluorescence intensity of each cell using the ROI Intensity Evolution plugin on ICY software.

\section{Plaque formation assay}

Acanthamoeba castellanii (ATCC 1034) was cultured at $30{ }^{\circ} \mathrm{C}$ in flasks with $20 \mathrm{ml}$ of PYG medium (peptone proteose $2 \%$, yeast extract $0.1 \%$, Na Citrate $2 \mathrm{H}_{2} \mathrm{O} 0.1 \%, \mathrm{CaCl}_{2} 0.4 \mathrm{mM}$, $\mathrm{MgSO}_{4} 4 \mathrm{mM}, \mathrm{Na}_{2} \mathrm{HPO}_{4} 2.5 \mathrm{mM}, \mathrm{KH}_{2} \mathrm{PO}_{4} 2.5 \mathrm{mM}$, Fe( $\left(\mathrm{NH}_{4}\right)_{2}\left(\mathrm{SO}_{4}\right)_{2} 0.05$ mM Glucose 0.1 M). 100 $\mu \mathrm{l}$ of overnight bacterial culture were pelleted by centrifugation at $4000 \mathrm{rpm}$ for $5 \mathrm{~min}$ and resuspended in $1 \mathrm{ml}$ of $\mathrm{M} 63$ medium. $100 \mu \mathrm{l}$ of this resuspension at $\mathrm{OD}_{600 \mathrm{~nm}}$ of 0.1 were spread on M63 Glucose $0.2 \%$ agar plates to form a bacterial lawn. The plates were dried $20 \mathrm{~min}$. Amoeba's cells were collected by centrifugation, washed once with M63 medium, and different number of amoebae cell in $5 \mu \mathrm{l}$ M63 were deposited on the top of the agar plate. Plates were incubated at $30^{\circ} \mathrm{C}$ for 5 days. The least number of Acanthamoeba castellanii cells deposited above that was able to form plaque on the bacterial lawns was defined as the minimum number of cells required for plaque formation in this study.

Infections of Galleria mellonella

257

258

259

260

Infections of Galleria larvae were done as previously described [36] with some modifications. The bacterial dose of approx. 60000 bacteria/injection was evaluated in preliminary experiment as such to obtain larvae mortality within $40 \mathrm{~h}$ post-pricking. Incubations were done at $30{ }^{\circ} \mathrm{C}$ and larvae were counted every $45 \mathrm{~min}$. The death was evaluated by the insusceptibility to touch by plastic tweezers. The dead larvae were removed from the dish. The experiment was repeated three times with 20 larvae used for each condition.

\section{Drosophila oral infections}

Drosophila melanogaster white Canton-S $\left(w^{\mathrm{CS} 10}\right)$ flies were maintained at $25^{\circ} \mathrm{C}$. Oral infection was done on 4 to 8-day old female flies let starving in an empty vial for 3-4 $\mathrm{h}$ before being transferred to an infected vial containing bacterial solutions. P. chlororaphis PA23 (wild-type and the $\triangle$ exIBA mutant) and $P$. entomophila L48 (wild-type and exIA mutant) were grown in LB at $28-30{ }^{\circ} \mathrm{C}$. Overnight bacterial cultures were pelleted and suspended into a sterile $1 \%$ 
sucrose solution and adjusted to an $\mathrm{OD}_{600}$ of 100 corresponding to $6.6 \times 10^{10}$ bacteria $/ \mathrm{ml} .200$

$\mu \mathrm{l}$ of bacterial suspension was added to a filter paper disk on the top of a standard fly-feeding medium in the infected vials [37]. Flies were maintained at $29{ }^{\circ} \mathrm{C}$ and the survival was monitored over 2 weeks. Vials containing filter paper imbedded with $200 \mu 1 \%$ sucrose alone were used as negative control. Infection experiment was repeated three times with $40-50$ flies per conditions tested, only one representative experiment is shown. The statistical analysis was performed with SigmaPlot software using a log rank statistic method.

\section{Lipid raft isolation}

Epithelial cell line A549 was seeded in P100 flasks at $2 \times 10^{7}$ cells/flask. Thirty minutes before infection, $7 \mathrm{ml}$ of fresh DMEM media was added per plate, four plates were used per condition. Overnight bacterial cultures were diluted in LB medium and let grown up to $\mathrm{OD}_{600}$ of 1.1 before being added on A459 monolayer cells at an $\mathrm{MOI}$ of 10 . Cells infected with $P$. chlororaphis PA23 wild-type and exIBA mutant were incubated at $30{ }^{\circ} \mathrm{C}$ in presence of $5 \% \mathrm{CO}_{2}$ for $3 \mathrm{~h}$ and 30 min. Cells infected with $P$. aeruginosa IHMA87 strain were incubated at $37^{\circ} \mathrm{C}$ in presence of 5 $\% \mathrm{CO}_{2}$ for $4 \mathrm{~h}$. Infection was monitored by microscopy and stopped when approximately $70 \%$ of the cells started to change the morphology and shrink. At the end of the infection, the culture medium was used for quantification of lactate dehydrogenase (LDH, Roche) to evaluate the level of cytotoxicity (cell permeabilization), while the infected A549 cells were washed twice with PBS and then resuspended into $1.6 \mathrm{ml}$ of $50 \mathrm{mM}$ HEPES, $150 \mathrm{mM} \mathrm{NaCl}, 5$ mM EDTA, 1 \% TritonX-100, pH7.4, containing Protease inhibitors cocktail (Complete, Roche). Resuspended A549 cells were incubated in ice for $1 \mathrm{~h}$ and kept in suspension by frequently vortexing. The debris were eliminated by low speed centrifugation at 1,000 g for $10 \mathrm{~min}$ at 4 ${ }^{\circ} \mathrm{C}$ and the supernatants were applied on a sucrose gradient. After ultracentrifugation at $40,000 \mathrm{rpm}$ for $16 \mathrm{~h}$ at $4{ }^{\circ} \mathrm{C}$, the lipid rafts are visible as a white cloudy band. Fractions of $1 \mathrm{ml}$ were recovered from the top to the bottom of each tubes and loaded on a gradient $4-12 \%$ SDS-PAGE Bis-Tris gel (BioRad). For Western Blot analysis, the proteins were transferred onto a PVDF membrane and incubated with mixture of rabbit polyclonal anti-ExIA antibodies. The mixture was composed by antibodies against ExIA-synthetic peptides diluted 500x [19], antibodies anti-ExIA-Cter and anti-ExIA-delta Cter both diluted 1 000x [25]. Mouse antiFlotillin-2 antibodies (BD Biosciences) were used as lipid raft marker at a dilution of 1:10,000. 


\section{Acknowledgments}

301

302

303

304

305

306

307

308

309

310

311

312

313

314

315

We thank Marie-Odile Fauvarque and Emmanuel Taillebourg (IRIG, BGE, CEA Grenoble) for help with Drosophila melanogaster experiments, and Isabelle Vallet-Gely for providing the $P$. entomophila mutant. We would like to thank Emma Chastaing for her technical assistance for the experiments with amoebas and fungi during her master internship (MAP, Lyon). The work was supported by grants from Agence Nationale de la Recherche (ANR-15-CE11-0018-01), the Laboratory of Excellence GRAL, financed within the University Grenoble Alpes graduate school (Ecoles Universitaires de Recherche) CBH-EUR-GS (ANR-17-EURE-0003) and the Fondation pour la Recherche Médicale (Team FRM 2017, DEQ20170336705).

\section{Figure Legends}

Figure 1. Genetic environment of the exIB-exIA operon. The sequences corresponding to $P$. chlororaphis PA23 (Pch), Pseudomonas aeruginosa PA7 (Pa) and Pseudomonas entomophila L48 $(P e)$ were retrieved from Pseudomonas genome database (www.pseudomonas.com) and annotated accordingly. The conserved genes exIA and exIB are in dark and light orange, respectively. Blue color indicates predicted putative regulators. Genes of unknown functions are in grey and encoding hypothetical proteins in white. Scale is shown.

Figure 2. Cytotoxicity of $\boldsymbol{P}$. chlororaphis PA23 on eukaryotic cells is ExIA-dependent. A. Kinetics of A549 epithelial cells cytotoxicity depends on MOI. Epithelial cells were infected with increasing quantities of bacteria ranging from $\mathrm{MOI}$ of 0.1 to 100 . B. P. chlororaphis is cytotoxic in vitro in an ExIBA-dependent manner. Epithelial cells (A549, upper panel) or murine macrophages (J774, lower panel) were infected at a $\mathrm{MOI}$ of 10 at $30^{\circ} \mathrm{C}$ with $P$. chlororaphis and $P$. aeruginosa strains, as indicated. Cell death was monitored by PI incorporation. Fluorescence emission at $590 \mathrm{~nm}$ of PI expressed as arbitrary units (A.U.) was recorded in function of time. Experiments were carried out in triplicates, and the error bars indicate the standard deviation.

Figure 3. Consequences of interactions between epithelial cells and P. chlororaphis PA23 or exIBA isogenic mutant. A. Epithelial cells A549 expressing cytoplasmic GFP were incubated with P. chlororaphis PA23 or exIBA mutant at $\mathrm{MOI}$ of 10. Incorporation of PI (red) into cell 
nuclei was followed by confocal videomicroscopy. Times post-infection are shown as "h:min". One z-position is represented. Scale bar shown on the last image corresponds to $20 \mu \mathrm{m}$. Images are representative of five positions simultaneously recorded for each condition. B. Intensities of GFP and PI were quantified on time lapse films reported in A. Five cells were analyzed in each condition. The GFP intensities are represented by solid lines and PI intensities by dashed lines, using the same color code for each selected cell.

\section{Figure 4. ExIA ${ }^{P c h}$ fractionates with lipid raft portion of infected epithelial cell membranes.}

A549 epithelial cells were infected with $P$. chlororaphis (wild-type or exIBA mutant) or $P$. aeruginosa IHMA87 wild-type strain, used as positive control at $\mathrm{OD}_{600}$ of 1.0 with an $\mathrm{MOI}$ of 10. The fractions with detergent-resistant membranes (DRM) containing lipid rafts, the detergent-soluble membranes (DSM) and the cytoplasm (C) were separated on sucrose gradient after treatment with $1 \%$ Triton X-100, as described in Material and Methods, and analyzed by Western blot using anti-ExIA antibodies mixture. Anti Flotillin-2 antibodies were used to localize DRM-containing fractions. Numbers on the top refers to the twelve fractions recovered from the top to the bottom of sucrose gradient, while sizes of proteins marker in (kDa) are shown on the right of the Western Blot.

Figure 5. ExIA is not involved in interaction of $\boldsymbol{P}$. chlororaphis PA23 with fungus Sclerotinia sclerotiorum and Botrytis cinerea nor in resistance to grazing by Acanthamoeba castellanii.

A. Radial diffusion assays to assess Sclerotinia sclerotiorum inhibition of growth in vitro on PDA plates with $P$. chlororaphis wild-type or $\triangle$ exIBA strains. $5 \mu$ of $P$. chlororaphis at $O_{600 \mathrm{~nm}}$ of 0.1 was deposited to the center of a $90 \mathrm{~mm}$ Petri dish inoculated with either plugs of Sclerotinia sclerotiorum or Botrytis cinerea mycelium. Plates were incubated at $25{ }^{\circ} \mathrm{C}$ until the mycelium covers the plate. Lengths of fungal inhibition zone was measured for 5 independent experiments. No statistical difference was measured between the strains (Mann-Whitney test). B. Acanthamoeba castellanii plaque formation assay. The plaques formed by amoebae on the lawns of $P$. chlororaphis wild-type or $\triangle$ exIBA strains. $P$. aeruginosa is used as a negative control since this bacterium kill Acanthamoeba. E. coli is used as a positive control since this bacterium can be phagocyted. The number of amoebae cells deposited on the plate is indicated (from $5.10^{4}$ on the top left to $5.10^{1}$ on the bottom right corner). $100 \mu$ l of bacterial 
358 culture at $\mathrm{OD}_{600}$ of 0.1 was spread onto each plate containing M63 nutrient agar with glucose $0.2 \%$.

Figure 6. ExIA contributes to killing of wax moth larva Galleria mellonella by $P$. chlororaphis

PA23. A. Representative experiment showing dead larvae after injection of $P$. chlororaphis

PA23. Note the appearance of a dark color as indication of melanization. B. Survival rates of the larvae are expressed as percentage (\%). The mortality was scored every $45 \mathrm{~min}$. Larvae were injected with approx. $6 \times 10^{4}$ bacteria in PBS, and let at $30^{\circ} \mathrm{C}$. Control larvae were injected with sterile PBS. Log rank test was used to determine statistically significant difference with $\mathrm{P}<0.001(* * *)$.

Figure 7. ExIA is important for Drosophila melanogaster oral infection by Pseudomonas sp.

4-8 days old female flies were fed with $1 \%$ sucrose containing $O D_{600}=100$ of indicated strains. In right panel infection with P. chlororaphis P23 (Pch) wild-type (in orange) or ex/BA mutant (in brawn); in left panel flies infection with P. entomophila L48 $(\mathrm{Pe})$ wild-type (dark green) or exIBA mutant (light green). Flies were incubated at $29^{\circ} \mathrm{C}$ and survival was monitored up to 10 days. A solution of $1 \%$ sucrose without bacteria was a control condition (grey line). Log rank test was used to determine statistically significant difference with $P<0.05(*)$ and $\mathrm{P}<0.001\left({ }^{* *}\right) . \mathrm{P}=0.0145$ (between $P c h$ and $P c h \Delta e x \mid B A$ ), $\mathrm{P}=0.0001$ (between $P e$ and Pe-ex/BA mut). 
1. Silby MW, Winstanley C, Godfrey SA, Levy SB, Jackson RW. Pseudomonas genomes: diverse and adaptable. FEMS microbiology reviews 2011;35(4):652-680.

2. Kupferschmied $\mathbf{P}$, Maurhofer $\mathbf{M}$, Keel $\mathbf{C}$. Promise for plant pest control: root-associated pseudomonads with insecticidal activities. Frontiers in plant science 2013;4:287.

3. Silby MW, Cerdeno-Tarraga AM, Vernikos GS, Giddens SR, Jackson RW et al. Genomic and genetic analyses of diversity and plant interactions of Pseudomonas fluorescens. Genome biology 2009;10(5):R51.

4. Wu X, Monchy S, Taghavi S, Zhu W, Ramos J et al. Comparative genomics and functional analysis of niche-specific adaptation in Pseudomonas putida. FEMS microbiology reviews 2011;35(2):299-323.

390 5. Organization WH. Guidelines for the prevention and control of carbapenem-resistant 391 Enterobacteriaceae, Acinetobacter baumannii and Pseudomonas aeruginosa in health care facilities. 392 World Health Organization 2019.

393 6. Manuel J, Selin C, Fernando WG, de Kievit T. Stringent response mutants of Pseudomonas 394 chlororaphis PA23 exhibit enhanced antifungal activity against Sclerotinia sclerotiorum in vitro. 395 Microbiology (Reading, England) 2012;158(Pt 1):207-216.

396 7. Nandi M, Selin C, Brassinga AK, Belmonte MF, Fernando WG et al. Pyrrolnitrin and Hydrogen Cyanide Production by Pseudomonas chlororaphis Strain PA23 Exhibits Nematicidal and Repellent Activity against Caenorhabditis elegans. PloS one 2015;10(4):e0123184.

399 8. Savchuk S, Dilantha Fernando WG. Effect of timing of application and population dynamics on

400

401

402

403

404

405

406

407

408

409

410

411

412

413

414

415

416

417

418

419

420

421

422

423

424

425

426

427 the degree of biological control of Sclerotinia sclerotiorum by bacterial antagonists. FEMS microbiology ecology 2004;49(3):379-388.

9. Flury $\mathbf{P}$, Aellen N, Ruffner B, Pechy-Tarr $\mathbf{M}$, Fataar $\mathbf{S}$ et al. Insect pathogenicity in plantbeneficial pseudomonads: phylogenetic distribution and comparative genomics. The ISME journal 2016;10(10):2527-2542.

10. Nandi M, Selin C, Brawerman G, Fernando WG, de Kievit TR. The global regulator ANR is essential for Pseudomonas chlororaphis strain PA23 biocontrol. Microbiology (Reading, England) 2016;162(12):2159-2169.

11. Winsor GL, Griffiths EJ, Lo R, Dhillon BK, Shay JA et al. Enhanced annotations and features for comparing thousands of Pseudomonas genomes in the Pseudomonas genome database. Nucleic acids research 2016;44(D1):D646-653.

12. Loewen PC, Villenueva J, Fernando WG, de Kievit T. Genome Sequence of Pseudomonas chlororaphis Strain PA23. Genome announcements 2014;2(4).

13. Pechy-Tarr M, Bruck DJ, Maurhofer M, Fischer E, Vogne $C$ et al. Molecular analysis of a novel gene cluster encoding an insect toxin in plant-associated strains of Pseudomonas fluorescens. Environmental microbiology 2008;10(9):2368-2386.

14. Ruffner B, Pechy-Tarr M, Hofte M, Bloemberg G, Grunder J et al. Evolutionary patchwork of an insecticidal toxin shared between plant-associated pseudomonads and the insect pathogens Photorhabdus and Xenorhabdus. BMC genomics 2015;16:609.

15. Molina-Santiago C, Pearson JR, Navarro Y, Berlanga-Clavero MV, Caraballo-Rodriguez AM et al. The extracellular matrix protects Bacillus subtilis colonies from Pseudomonas invasion and modulates plant co-colonization. Nature communications 2019;10(1):1919.

16. Vacheron J, Pechy-Tarr M, Brochet S, Heiman CM, Stojiljkovic M et al. T6SS contributes to gut microbiome invasion and killing of an herbivorous pest insect by plant-beneficial Pseudomonas protegens. The ISME journal 2019;13(5):1318-1329.

17. Dorosky RJ, Pierson LS, 3rd, Pierson EA. Pseudomonas chlororaphis Produces Multiple RTailocin Particles That Broaden the Killing Spectrum and Contribute to Persistence in Rhizosphere Communities. Applied and environmental microbiology 2018;84(18). 
18. Dorosky RJ, Yu JM, Pierson LS, 3rd, Pierson EA. Pseudomonas chlororaphis Produces Two Distinct R-Tailocins That Contribute to Bacterial Competition in Biofilms and on Roots. Applied and environmental microbiology 2017;83(15).

19. Elsen S, Huber P, Bouillot S, Coute $\mathbf{Y}$, Fournier $\mathbf{P}$ et al. A type III secretion negative clinical strain of Pseudomonas aeruginosa employs a two-partner secreted exolysin to induce hemorrhagic pneumonia. Cell host \&amp; microbe 2014;15(2):164-176.

20. Reboud E, Basso P, Maillard AP, Huber P, Attree I. Exolysin Shapes the Virulence of Pseudomonas aeruginosa Clonal Outliers. Toxins 2017;9(11).

21. Vodovar N, Vallenet D, Cruveiller S, Rouy Z, Barbe V et al. Complete genome sequence of the entomopathogenic and metabolically versatile soil bacterium Pseudomonas entomophila. Nature biotechnology 2006;24(6):673-679.

22. Basso P, Wallet P, Elsen S, Soleilhac E, Henry T et al. Multiple Pseudomonas species secrete exolysin-like toxins and provoke Caspase-1-dependent macrophage death. Environmental microbiology 2017;19(10):4045-4064.

23. Basso P, Ragno M, Elsen S, Reboud E, Golovkine $G$ et al. Pseudomonas aeruginosa PoreForming Exolysin and Type IV Pili Cooperate To Induce Host Cell Lysis. mBio 2017;8(1).

24. Reboud E, Bouillot S, Patot S, Beganton B, Attree I et al. Pseudomonas aeruginosa ExIA and Serratia marcescens ShlA trigger cadherin cleavage by promoting calcium influx and ADAM10 activation. PLoS pathogens 2017;13(8):e1006579.

25. Berry A, Han K, Trouillon J, Robert-Genthon M, Ragno M et al. cAMP and Vfr Control Exolysin Expression and Cytotoxicity of Pseudomonas aeruginosa Taxonomic Outliers. Journal of bacteriology 2018;200(12).

26. Anderson JA, Staley J, Challender M, Heuton J. Safety of Pseudomonas chlororaphis as a gene source for genetically modified crops. Transgenic research 2018;27(1):103-113.

27. Shen X, Wang Z, Huang X, Hu H, Wang W et al. Developing genome-reduced Pseudomonas chlororaphis strains for the production of secondary metabolites. BMC genomics 2017;18(1):715.

28. Montana S, Lazzaro T, Uong S, Place K, Iriarte A et al. Genomics helps to decipher the resistance mechanisms present in a Pseudomonas chlororaphis strain recovered in an HIV patient. New microbes and new infections 2018;25:45-47.

29. Keel C. A look into the toolbox of multi-talents: insect pathogenicity determinants of plantbeneficial pseudomonads. Environmental microbiology 2016;18(10):3207-3209.

30. Matz C, Moreno AM, Alhede M, Manefield M, Hauser AR et al. Pseudomonas aeruginosa uses type III secretion system to kill biofilm-associated amoebae. The ISME journal 2008;2(8):843-852.

31. Pukatzki S, Kessin RH, Mekalanos JJ. The human pathogen Pseudomonas aeruginosa utilizes conserved virulence pathways to infect the social amoeba Dictyostelium discoideum. Proceedings of the National Academy of Sciences of the United States of America 2002;99(5):3159-3164.

32. Vodovar N, Vinals M, LiehI P, Basset A, Degrouard J et al. Drosophila host defense after oral infection by an entomopathogenic Pseudomonas species. Proceedings of the National Academy of Sciences of the United States of America 2005;102(32):11414-11419.

33. Opota O, Vallet-Gely I, Vincentelli R, Kellenberger C, lacovache I et al. Monalysin, a novel sspore-forming toxin from the Drosophila pathogen Pseudomonas entomophila, contributes to host intestinal damage and lethality. PLoS pathogens 2011;7(9):e1002259.

34. Vallet-Gely I, Novikov A, Augusto L, LiehI P, Bolbach $\mathbf{G}$ et al. Association of hemolytic activity of Pseudomonas entomophila, a versatile soil bacterium, with cyclic lipopeptide production. Applied and environmental microbiology 2010;76(3):910-921.

35. Golovkine G, Faudry E, Bouillot S, Elsen S, Attree I et al. Pseudomonas aeruginosa Transmigrates at Epithelial Cell-Cell Junctions, Exploiting Sites of Cell Division and Senescent Cell Extrusion. PLoS pathogens 2016;12(1):e1005377.

36. Sentausa E, Basso P, Berry A, Adrait A, Bellement $\mathbf{G}$ et al. Insertion sequences drive the emergence of a highly adapted human pathogen. Microbial genomics 2019. 
bioRxiv preprint doi: https://doi.org/10.1101/807867; this version posted October 17, 2019. The copyright holder for this preprint (which was not certified by peer review) is the author/funder. All rights reserved. No reuse allowed without permission. a signalling molecule controls Pseudomonas entomophila virulence. Cellular microbiology 2010;12(11):1666-1679.

481 

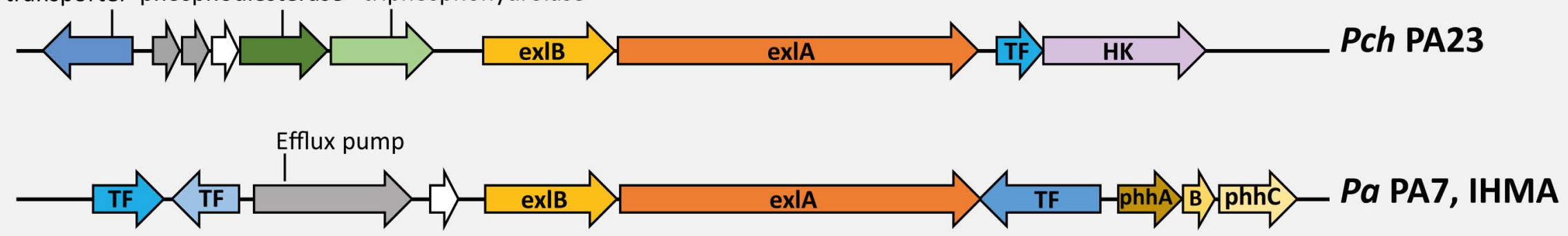

Isoquinoline 1-oxidoreductase

cytocrome $\mathrm{c}$ subunit $\alpha$ subunit $\beta$

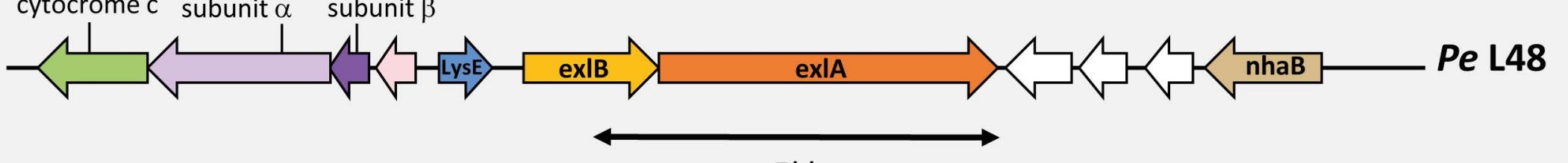



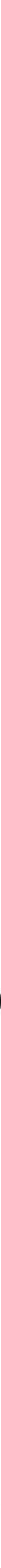

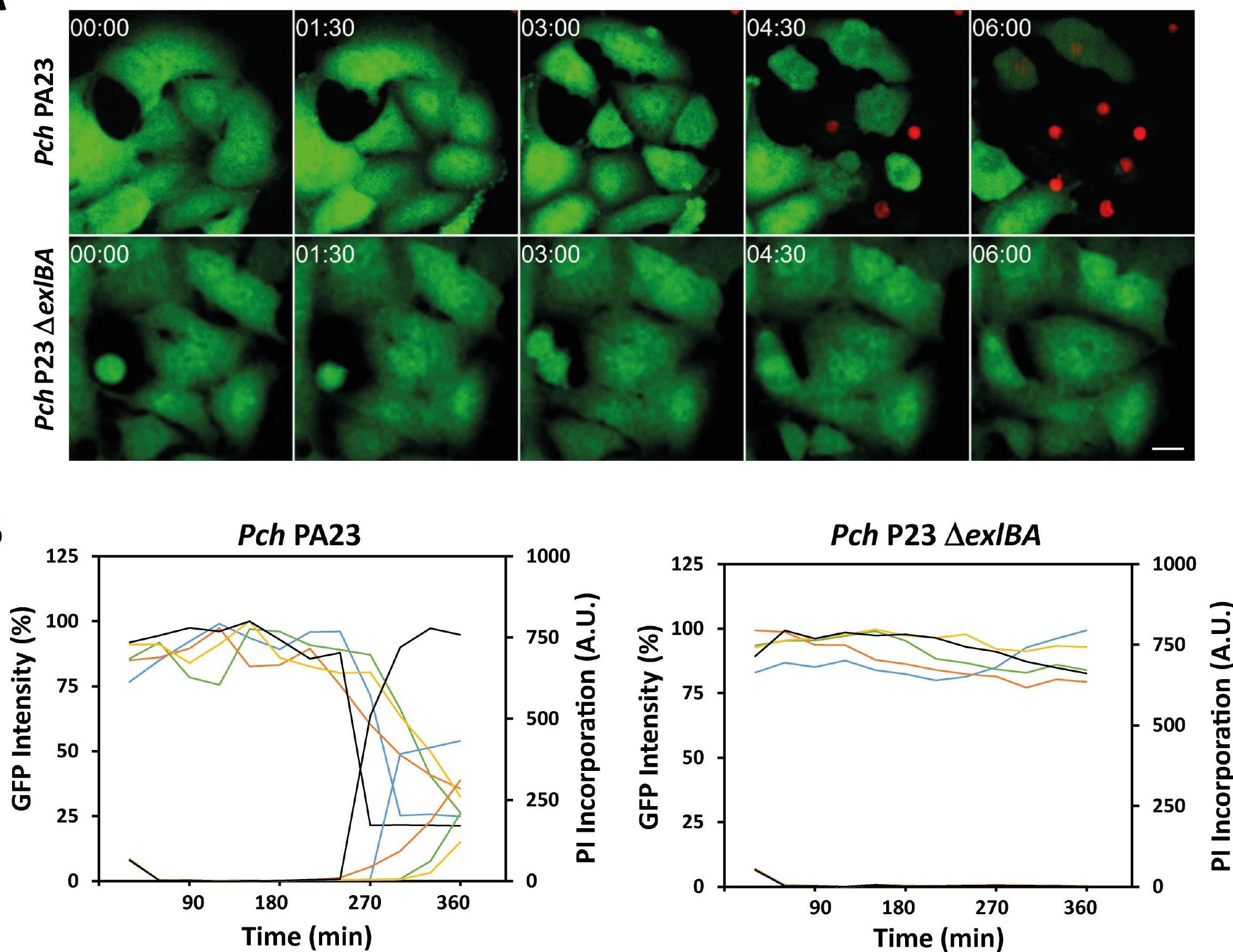

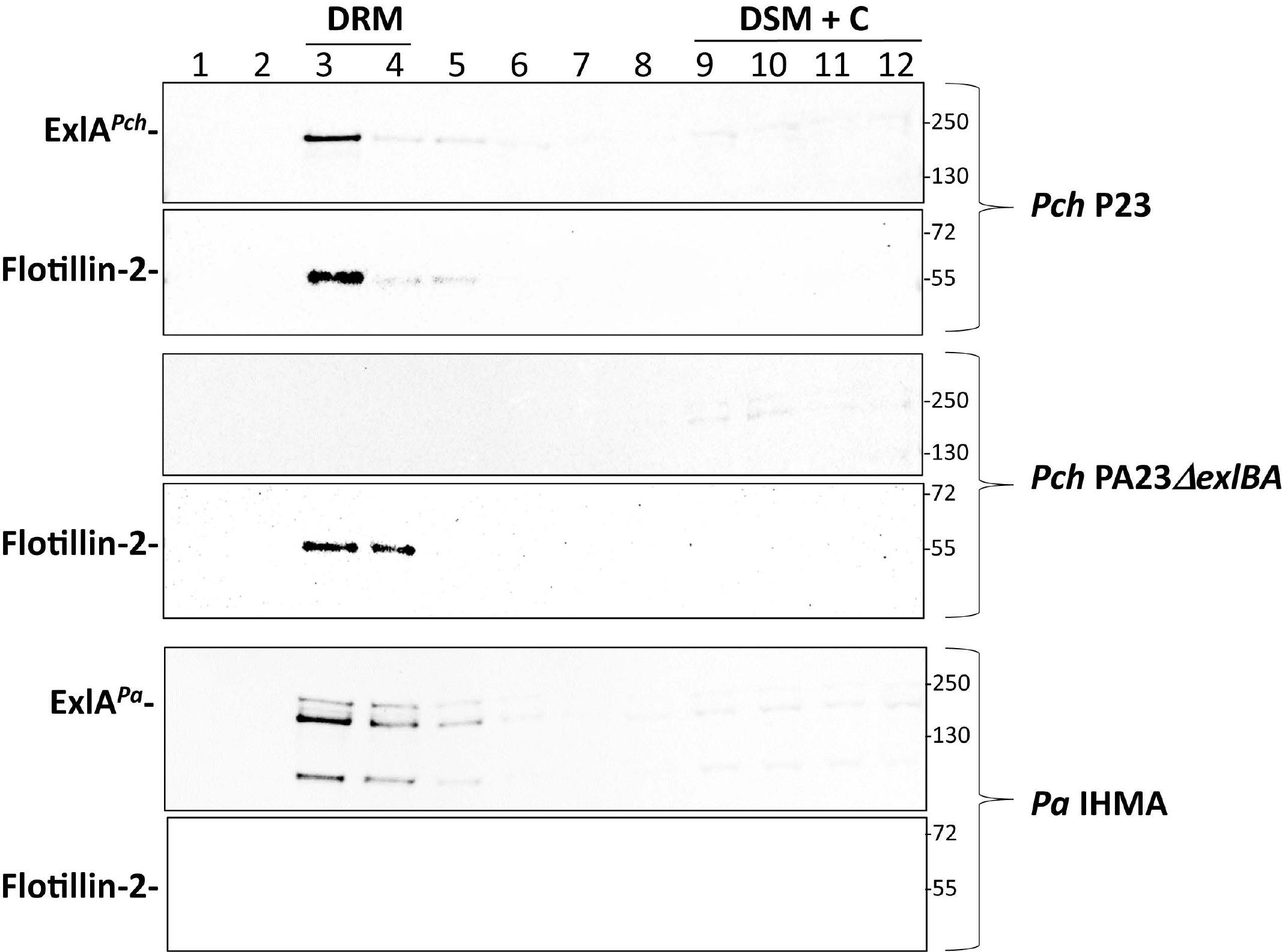

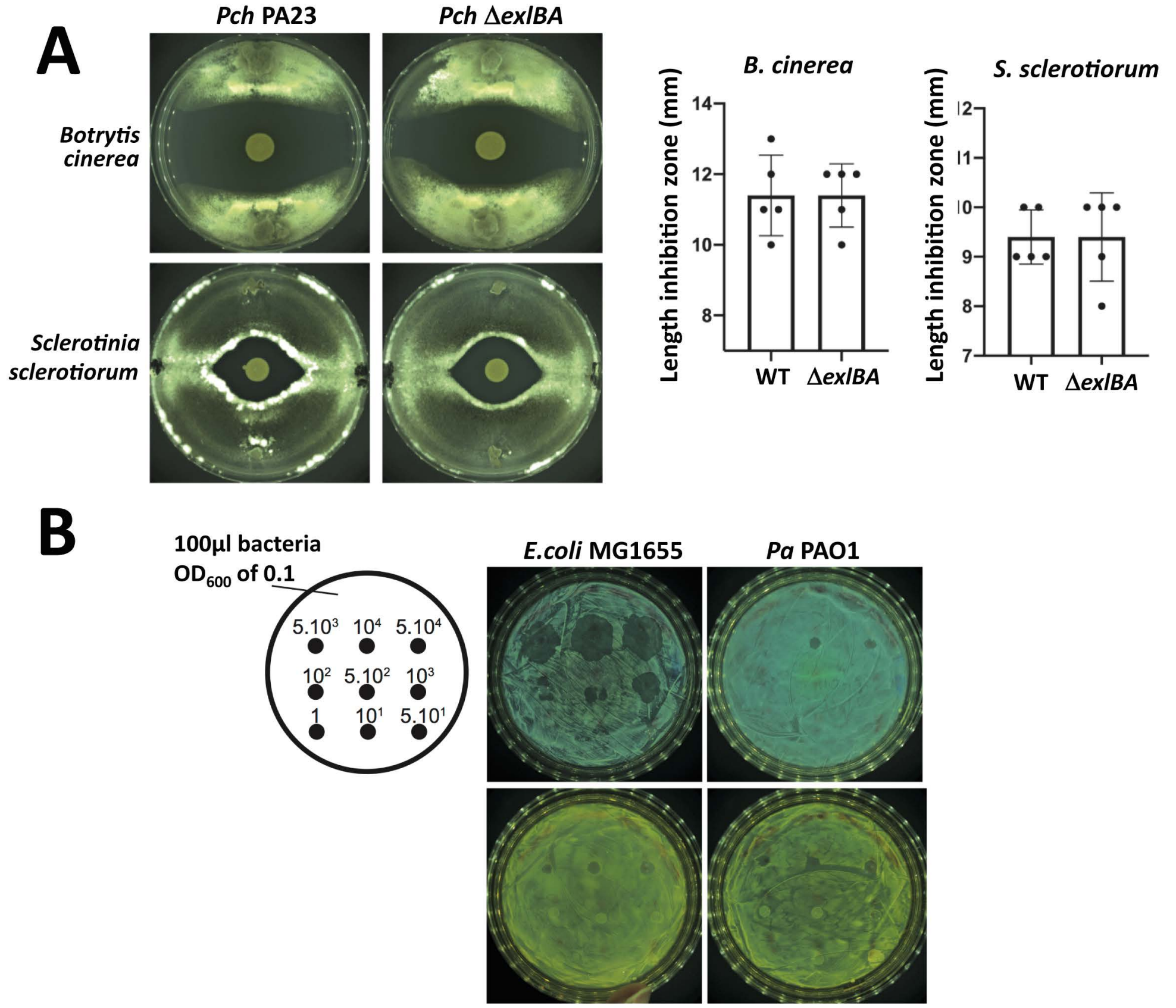

Pch PA23

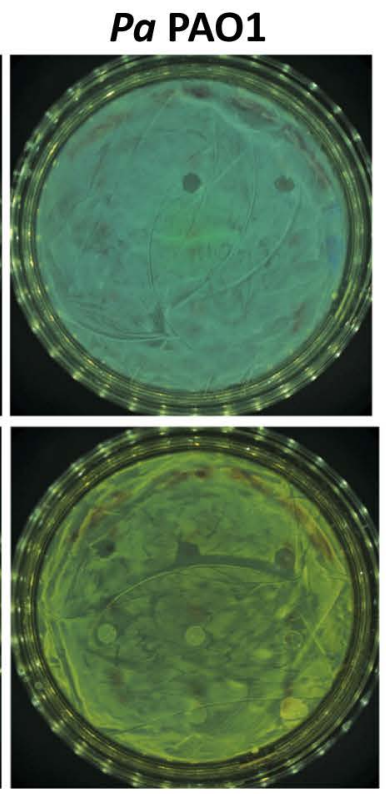

Pch $\triangle e x / B A$ 


\section{Pch PA23}

Pch PA23 4 exIBA

B
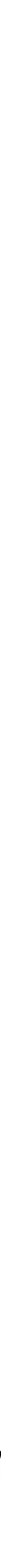

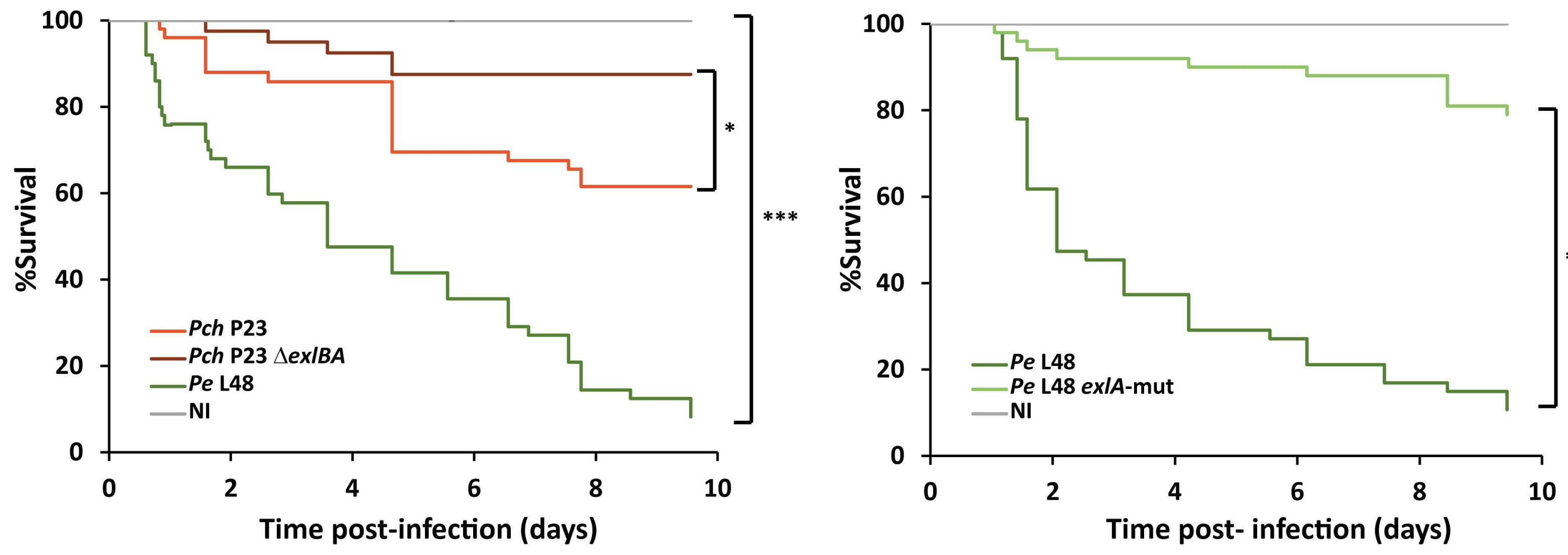